\title{
A Carcinogenic metabolism induced by a deficiency of the endocrine pancreas
}

\author{
Maurice Israël* \\ CNRS Gif sur Yvette and 2 Av. Aristide Briand 91440 Bures sur Yvette, France
}

\begin{abstract}
An overview of cancer metabolism is briefly presented. Apparently, differentiated tissues resistant to insulin but sensitive to catabolic hormones become nutritional reservoirs for populations of stem cells that display at the enzymatic level a hybrid response to both insulin and glucagon. This leads them to rewire their metabolic pathways into a "carcinogenic mode", giving them a selective advantage favoring their mitotic development. Normally, when pancreatic beta cells release insulin they co-release GABA that turns off glucagon release from alpha cells, but also terminates the release of insulin by closing its release mechanism. Hence, a GABA deficiency will induce a chronic insulin release that renders differentiated cells resistant to anabolic insulin, while sensitive to glucagon. In contrast, new mitotic stem cells that have not been submitted to desensitization, display a dual sensitivity to insulin and glucagon, no longer blocked by the release of GABA. In these stem cells glycolysis increases via insulin effects, but pyruvate kinase and pyruvate dehydrogenase are inhibited via glucagon action. This "bottle neck" at the last step of glycolysis will be overcome by a rewiring of metabolic pathways. The GABA deficiency also increases epinephrine release that blocks somatostatin release from delta cells, enhancing the effect of growth hormone on lipolysis and diacylglycerol production. The latter stimulates protein kinase $\mathrm{C}$, and the formation of a phosphatase inhibitor, which maintains the glycolytic "bottle neck" and a metabolic rewiring typical of cancer.
\end{abstract}

\begin{abstract}
Abbreviations: PKA: Protein Kinase A; PKB: Protein Kinase B; PKC: Protein Kinase C; IGF: Insulin Like Growth Factor; MAPK: Mitogen-Activated Protein Kinase Pathway; PI3 Kinase: Phosphatidylinositide 3-Kinase Pathway; PDK: Phosphoinositide-Dependent Protein Kinase, PK: Pyruvate Kinase; PDH: Pyruvate Dehydrogenase; ATGL: Adipose triglyceride lipase; PEPCK: Phosphoenolpyruvate Carboxykinase; ACC: Acetyl CoA Carboxylase; PDE: Phosphodiesterase; CPI- 17: C-Kinase-Activated Protein Phosphatase-1 (PP1) Inhibitor; I1: PP1 Phosphatase Inhibitor; GABA: Gamma Aminobutyric Acid; cAMP: Cyclic AMP; OAA: Oxaloacetate; PEP: Phosphoenolpyruvate; DAG: Diacyl Glycerol; IP3: Inositol 1,4,5P; PIP2: Phosphatidyl Inositol $(4,5)$ Bis P; PIP3: Phosphatidylinositol $(3,4,5) \mathrm{P}$.
\end{abstract}

\section{Introduction}

In cancer, metabolic pathways are rearranged in a very special way that confers to tumor cells a selective advantage, enabling them to plunder nutrients from tissue stores and ensure the mitotic development of the tumor $[1,2]$. The main feature of tumor cell metabolism is the avid uptake of glucose while their glycolytic pathway gets interrupted at the pyruvate kinase (PK) step. In cancer, it is the $\mathrm{M} 2$ form of $\mathrm{PK}$ that prevails [3]. In addition, pyruvate dehydrogenase $(\mathrm{PDH})$ is also inhibited by phosphorylation [4] closing the glycolytic source of acetyl CoA at the entry of the citric acid cycle. It is like if tumor cells had received an insulin message eliciting the entry of glucose and glycolysis, paradoxically associated to an inhibition of PK and PDH by phosphorylation as one observes when glucagon or epinephrine are secreted to elicit neoglucogenesis and interrupt glycolysis $[5,6]$. This interruption of the glycolytic pathway is overcome by a special metabolic rewiring process. The phosphoenolpyruvate (PEP), which is no longer converted by PK into pyruvate will rather feed the enzyme phosphoenolpyruvate carboxykinase (PEPCK) and form oxaloacetate (OAA). The condensation of OAA and acetyl CoA will then form citric acid starting the Krebs cycle. But since PDH is also blocked by phosphorylation, acetyl CoA will essentially be provided through the beta oxidation of fatty acids, forming ketone bodies in the liver. These ketone bodies will give back acetyl CoA in tumor cells and feed their citrate condensation reaction. Tumor cells depend of this source of acetyl CoA because their own beta oxidation is inhibited by the malonyl CoA intermediate formed by acetyl CoA carboxylase (ACC) along their active fatty acid synthesis pathway, required for making new membranes of dividing cells. We indeed know that malonyl CoA inhibits the mitochondrial fatty acid carnityl transporter and thus their mitochondrial beta oxidation. With OAA coming from PEP and acetyl CoA coming from ketone bodies the citrate condensation starts the Krebs cycle of tumor cells, citrate is formed but readily quits the mitochondria to give back in the cytosol acetyl CoA and OAA, via ATP citrate lyase. Acetyl CoA will feed (ACC) and the fatty acid synthesis route forming the malonyl CoA intermediate; as for OAA it supports tumor cells transaminations that recycle the amines coming from the elevated amino acid supply. These are essentially provided by an increased proteolysis in muscles. Alanine is for example transaminated into pyruvate and the latter converted to lactate that increases in cancer, as initially observed by Warburg [7,8]. Other features include: the truncated urea cycle (argininosussinate synthase is inhibited) [9]; the truncated Krebs cycle and supply of alpha ketoglutarate in relation to the mitochondrial shuttles; the polyamine metabolism; the supply of nucleotide bases or the pentose pathway, all providing molecular building blocks for making new cells [2,10-12]. Here, we

Correspondence to: Maurice Israël, CNRS Gif sur Yvette and 2 Av. Aristide Briand 91440 Bures sur Yvette, France, E-mail: mauisrael@wanadoo.fr

Key words: cancer metabolism; GABA; glucagon; insulin; PKC; stem cells

Received: August 03, 2017; Accepted: August 30, 2017; Published: September 04,2017 
give an overview of cancer metabolism focused on the mechanism that controls the metabolic rewiring process.

\section{The PK and PDH glycolytic bottle neck of tumor cells, a failure of phosphatases?}

The effect of catabolic hormones glucagon and epinephrine mediated by Gs coupled protein receptors (GPCR) triggers the production of glucose. Adenylate cyclase is stimulated, cAMP increases and activates PKA, which leads to the phosphorylation on tyrosine for $\mathrm{PK}$ and serine for $\mathrm{PDH}$ via their respective kinases and to the inhibition of these enzymes, closing the glycolytic direction while opening the opposite glucose synthesis route [2]. In striated muscles this gluconeogenic message is cancelled; presumably, muscles have to preserve their glycolytic energy in order to escape from predators. This muscle exception may give some indications on the dephosphorylation process of $\mathrm{PK}$ and $\mathrm{PDH}$ that seems to fail in tumor cells. Muscle activity is associated to electrical charge moving along $\mathrm{T}$ tubules, until ryanodine receptors of endoplasmic reticulum sacs in the muscle depth. These sacs release calcium in the cytosol, which initiates the contraction. The increase of cytosolic calcium activates a phosphatase PP2B (calcineurin), which dephosphorylates an inhibitor I1 that locked another phosphatase PP1. The activation of PP1 may then dephosphorylate $\mathrm{PDH}$ and activate a tyrosine phosphatase that will dephosphorylate $\mathrm{PK}$; which opens back the glycolytic pathway in muscles. Moreover, calcium stimulates a phosphodiesterase (PDE) that hydrolyses cAMP into AMP; this cancels the inhibition by CAMP of fructose 2-6 bisphosphate synthesis, this allosteric activator of phosphofructokinase 1 increases and boosts glycolysis, preserving muscle glycolytic activity. In addition, muscle fibers have presumably more epinephrine sensitive GPCRs of the Go or Gi type rather than Gs, attenuating the production and inhibitory effect of cAMP on the synthesis of fructose 2-6 bis phosphate. In sum, we may suspect that a similar dephosphorylation mechanism is altered in tumor cells [10].

Another example gives even more pertinent indications on the failure of PK and PDH dephosphorylation in tumor cells. Normally, when anabolic insulin is released it activates tyrosine kinase receptors. The latter stimulate the MAP kinase mitogenic cascade and PI3 kinase survival pathways (remember that they are known targets for most oncogenes). PI3 Kinase phosphorylates membrane phosphoinositol PIP2 into PIP3 recruiting PKB in the membrane where it is activated by PDK (a membrane kinase). PKB has effects that are opposite to PKA; it inhibits a set of protein kinases and activates phosphatases leading to the dephosphorylation of enzymes such as $\mathrm{PK}$ and $\mathrm{PDH}$ that were phosphorylated via catabolic hormones. As was the case for the muscle exception, calcium seems to play again a prominent role. Indeed, a downstream effect of the insulin receptor activation is the stimulation of a phospholipase $\mathrm{C}$ that hydrolyses PIP2 into diacylglycerol (DAG) and inositol 1,4,5 phosphate (IP3). This compound binds IP3 receptors of the endoplasmic reticulum eliciting the release of calcium in the cytosol. If DAG is consumed, calcium may act as for the muscle exception and elicit the dephosphorylation of $\mathrm{PK}$ and $\mathrm{PDH}$ via PP1 phosphatase activation. It also stimulates a PDE that hydrolyses cAMP, which increases fructose 2-6 bis $\mathrm{P}$ the allosteric activator of glycolysis. But here the role of DAG seems to control the system, when it increases it will activate PKC and this changes the situation. PKC stimulates the synthesis of another inhibitor of PP1 known as CPI-17, which then maintains the phosphorylation of PK and PDH. This closes the glycolytic source of acetyl CoA, and opens the fatty acid source of acetyl CoA via their beta oxidation splitting $[10,13]$. This is helped by an elevated AMP that stimulates AMP kinase, a known inhibitor of ACC [14]; fatty acids synthesis is delayed. Indeed, the malonyl CoA intermediate being low, fatty acid transport in mitochondria takes place, and they are split by beta oxidation into acetyl CoA. Then, the activation of PKC by DAG gradually activates AMP deaminase [15] and 5 ' nucleotidase [16], which decrease AMP. This cancels the activation of AMP kinase and its inhibitory effect on ACC [14], the synthesis of fatty acid becomes operational, malonic CoA increases, the beta oxidation of fatty acids is inhibited, the anabolic action of insulin fully develops. It is then necessary to reestablish the glycolytic source of acetyl CoA and to dephosphorylate $\mathrm{PK}$ and $\mathrm{PDH}$ by removing the CPI-17 inhibitor from PP1. Well the decrease of DAG consumed by the synthesis of lipids induced by insulin will cancel the activation of PKC by DAG and the formation of the PP1 inhibitor. This last step seems to fail in tumor cells since they maintain their PK and PDH phosphorylation and the glycolytic bottle neck, as if DAG failed to decrease [10]. This is pertinent to consider since we know that carcinogenic phorbol esters will also stimulate PKC. It is thus interesting to find why the activation of PKC by DAG is maintained, in relation the metabolic rewiring that takes place in tumor cells.

\section{A chronic failure of the endocrine pancreas explains the carcinogenic rewiring of metabolism}

The following observations are to be considered: In cancer, differentiated cells in liver, adipose tissue or muscles, respond to catabolic hormones (glucagon epinephrine and cortisol) depleting their stores of glycogen fat and proteins, for providing nutrients: glucose, amino acids and ketone bodies to tumor cells. As if differentiated cells, were selectively sensitive to catabolic hormones mobilizing their stores and became relatively resistant to anabolic insulin or IGF. The phosphorylation and inhibition of enzymes such as $\mathrm{PK}$ or $\mathrm{PDH}$ is related to their catabolic metabolism. The resistance to insulin is rather frequent; it is associated to diabetes type 2, to the metabolic syndrome, to obesity with associated leptin receptor desensitization. All these pathologies are believed to increase the incidence of cancer. On the other hand we know that tumor cells avidly take up glucose and nutrients, and actively synthesize lipids and proteins, forming new molecular building blocks for mitotic cells. Their response to insulin, IGF and growth hormone is evidently anabolic. However, this is not so simple, since the effects of catabolic hormones on enzymes such as $\mathrm{PK}$ and $\mathrm{PDH}$ persists in tumor cells, these enzymes are indeed phosphorylated and inhibited. This leads to a complete rewiring of metabolic pathways that we briefly recalled in the introduction. The question is to find out why the mitotic tumor cells display this dual response to anabolic and catabolic hormones? In every organ there are populations of stem cells that are committed to replace dead cells. Normally, when stem cells divide one daughter cell differentiates for replacing a dead cell, while the other gives back a stem cell. It is probable that the new daughter cells have not been submitted to the chronic process that desensitizes the insulin receptors of differentiated cells rendered resistant to insulin. Thus, new mitotic cells will be sensitive to both anabolic actions of insulin and catabolic actions of glucagon or epinephrine; the catabolic effect elicits the phosphorylation and inhibition of $\mathrm{PK}$ and $\mathrm{PDH}$. This hybrid metabolic response at the enzymatic level is overcome by a metabolic rewiring of pathways. This new metabolic rearrangement enables stem cells to plunder nutritional stores, the advantage they gain over differentiated cells, blocks their own differentiation, increasing their mitotic 
capabilities. The explanation we propose for their dual response to insulin and glucagon was inspired by the physiology of the endocrine pancreas $[17,18]$.

The beta cells of the endocrine pancreas secrete insulin but coreleases GABA. The action of GABA on metabotropic GABA B auto-receptors of beta cells is to terminate the release of insulin, and completely turn off its release. If GABA gets deficient there will be a small but persistent release of insulin that will in the long run, desensitize insulin receptors, and presumably leptin receptors, favoring diseases such as diabetes type 2 , metabolic syndrome or obesity. This concerns differentiated cells but not the newly formed mitotic stem cells that have not been submitted to this chronic desensitization process. The other role of GABA is also to block GABA A ionotropic receptors found on alpha and delta cells of the pancreas, inhibiting the release of glucagon and somatostatin respectively. Thus, when anabolic insulin is released, catabolic glucagon is blocked. In this way, anabolism excludes catabolism. However, if GABA gets deficient one may expect that insulin will be released while glucagon is not turned off. Mitotic stem cells that are not resistant to insulin and sensitive to glucagon may then display a hybrid response, their anabolism is activated, but PK and PDH remain phosphorylated as for catabolism; leading them to rewire their metabolism in the "carcinogenic mode" described. This is still not sufficient to fully describe the system, because insulin that activates via IP3 the release of calcium in the cytosol should have been able to activate phosphatases that dephosphorylate these enzymes, as was discussed above for the muscle exception or for IP3-calcium effects. The explanation we propose for the maintained inhibition of phosphatases is a persistent activation of PKC and CPI17 phosphatase inhibitor production, provoked by an elevated DAG concentration that stimulates PKC. But what keeps DAG elevated in these mitotic cells? Well again, if the release of GABA gets deficient it will act at some distance on the adrenal medulla, where it boosts the release of epinephrine. The latter inhibits somatostatin release from pancreatic delta cells and does now the job for GABA on delta cells [5]. It will not be the same for alpha cells since epinephrine stimulates glucagon release. Hence, the decrease of somatostatin re-established by epinephrine will boost the effects of growth hormone and IGF [19]. We do know that growth hormone increases lipolysis [20]; presumably, adipose triglyceride lipase (ATGL) initiates the process and produces fatty acids and DAG, compensating the consummation of DAG by the lipid synthesis pathway. Since DAG is maintained or increased, the stimulation of PKC is not terminated, it will stimulate the formation of CPI-17 the PP1 inhibitor, blocking phosphatases able to dephosphorylate $\mathrm{PK}$ and $\mathrm{PDH}$, which closes the glycolytic supply of acetyl CoA. On the other hand, the activation of a PDE by IP3 and cytosolic calcium is not affected; this decreases cAMP, and increases fructose 2-6 bis phosphate stimulating glycolysis above the PK and PDH bottle neck. This additional effect of the GABA deficiency completes the metabolic picture, the increase of growth hormone and DAG stimulates PKC and closes in fine the glycolytic supply of acetyl CoA, maintaining the dependency of tumor cells toward a ketogenic supply for this compound. Presumably, carcinogenic phorbol esters that stimulate PKC act in the same way. The stem cells in such a situation will have to rewire their metabolic pathways "in the carcinogenic mode" they dependent of nutritional supplies, provided by differentiated cells responding to catabolic hormones, but the metabolic advantage they acquire will ensure their mitotic development.

\section{Conclusions}

In sum, the GABA deficiency at the endocrine pancreas explains 1- the catabolic sensitivity of differentiated cells resistant to insulin, 2- the dual response of stem cells to insulin and glucagon, 3- the increase of growth hormone and DAG -activation of PKC, which maintains the glycolytic PK and PDH bottle neck. The consequence is a rewiring of metabolic pathways that is typical for cancer.

\section{Conflict of interest}

The author has no conflict of interest to declare related to this project. The paper is not supported by any grants.

\section{References}

1. Israël M, Schwartz L (2011) The metabolic advantage of tumor cells. Mol Cancer 10 70. [Crossref]

2. Israël M (2014) Signaling and metabolism in cancer: endocrine pancreas deficiency and hybrid anabolism-catabolism, drugs that undo the process. Cancer Therapy 10: 1-12.

3. Mazurek S1, Eigenbrodt E (2003) The tumor metabolome. Anticancer Res 23: 11491154. [Crossref]

4. Madhok BM, Yeluri S, Perry SL, Hughes TA, Jayne DG (2010) Dichloroacetate induces apoptosis and cell-cycle arrest in colorectal cancer cells. Br J Cancer 102 1746-1752. [Crossref]

5. Israël M (2012a) A possible primary cause of cancer: deficient cellular interactions in endocrine pancreas. Mol Cancer 11: 63-68.

6. Israël M (2012b) A primary cause of cancer: GABA deficiency in endocrine pancreas. Cancer Therapy 8: 171-183.

7. Warburg O (1956) On the origin of cancer cells. Science 123: 309-314. [Crossref]

8. Warburg O (1956) On respiratory impairment in cancer cells. Science 124: 269-270 [Crossref]

9. Wheatley DN, Campbell E (2003) Arginine deprivation, growth inhibition and tumor cell death: Deficient utilization of citrulline by malignant cell. Br J Cancer 89: 573-6. [Crossref]

10. Israël M (2017) Altered controls transforming normal metabolism into carcinogenic. $J$ Cancer Prev Curr Res 7: 00228-33.

11. Bucay AH (2007) The biological significance of cancer; mitochondria as a cause of cancer and the inhibition of glycolysis with citrate as a cancer treatment. Medical Hypotheses 69: 826-828.

12. Icard P, Lincet H (2012) The central role of citrate in the metabolism of cancer cells Biochemical research 23: SI 31-37.

13. Hue L, Taegtmeyer H (2009) The Randle cycle revisited: a new head for an old hat. $A m$ J Physiol Endocrinol Metab 297: E578-591. [Crossref]

14. Park SH, Gammon SR, Knippers JD, Paulsen SR, Rubink DS, et al. (2002) Phosphorylation-activity relationships of AMPK and acetyl-CoA carboxylase in muscle. J Appl Physiol 92: 2475-82.

15. Hohl CM (1999) AMP deaminase in piglet cardiac myocytes: effect on nucleotide metabolism during ischemia. Am J Physiol 276(5Pt2): H 1502-10.

16. Node K, Kitakaze M, Minamino T, Tada M, Inoue M, et al. (1997) Activation of ecto5 '-nucleotidase by protein kinase $\mathrm{C}$ and its role in ischaemic tolerance in the canine heart. Br J Pharmacol 120: 273-281. [Crossref]

17. Braun M, Wendt A, Buchard K, Salehi A, Sewing S, et al. ( 2004) GABAB receptor activation inhibits exocytosis in rat pancreatic-beta-cells by G-protein-dependent activation of calcineurin. $J$ Pysiol 559(Pt2): 397-409.

18. Taneera J, Jin Z, Jin Y, Muhammed SJ, Zhang E, et al. (2012) Gamma Aminobutyric acid (GABA) signaling in human pancreatic islets is altered in type 2 diabetes. Diabetologia 55: 1985-1994.

19. Israël M (2014) Carcinogenesis and endocrine pancreas deficiency, a working frame for cancer prevention or treatment. Int J Cancer Oncol 2: 1-5.

20. Møller N, Jørgensen JO (2009) Effects of growth hormone on glucose, lipid, and protein metabolism in human subjects. Endocr Rev 30: 152-177. [Crossref]

Copyright: (C2017 Israël M. This is an open-access article distributed under the terms of the Creative Commons Attribution License, which permits unrestricted use, distribution, and reproduction in any medium, provided the original author and source are credited. 